\title{
Increasing age, minority ethnic origin, and lack of sufficient medical insurance decrease likelihood of treatment for depression in the elderly
}

Crystal S, Sambamoorthi U, Walkup T, et al. Diagnosis and treatment of depression in the elderly medicare population: predictors, disparities, and trends. J Am Geriatr Soc 2003;51:1718-28.

What is the prevalence of treated depression in the elderly and what socioeconomic factors affect their treatment?

METHODS

$\square$

Design: Prospective cohort study.

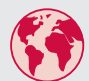

Setting: Medicare recipients, USA; 1992-98

क의

Population: 20966 Medicare Current Beneficiary Survey (MCBS) participants aged 65 and over, living in the community Exclusions: people enrolled in Medicare or MCBS for less than 1 year; people enrolled in Medicare risk health maintenance organisations.

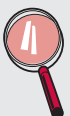

Assessment: Medicare claims were reviewed annually to identify people receiving a diagnosis of depression (ICD-9). Claims information and participant interviews were used to determine what, if any, treatment was received within that year (regardless of funding source). Information regarding participants' age, income, marital status, education, ethnicity, health status, functional ability, chronic medical conditions, and health insurance coverage (basic Medicare coverage only or plus supplemental coverage) was obtained.

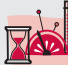

Outcomes: Diagnosis and treatment of depression.

Follow up period: Seven 1 year periods analysed.

\section{MAIN RESULTS}

The incidence of diagnosed depression increased significantly from $2.8 \%$ in 1992 to $5.8 \%$ in 1998 (OR 2.30, 95\% CI 1.91 to 2.77 ). Overall, $67.7 \%$ of those diagnosed with depression received treatment (of whom $47 \%$ received antidepressants only, $9.7 \%$ psychotherapy only, and $17.2 \%$ psychotherapy plus antidepressants). Racial/ethnic minorities (not including African Americans) were significantly less likely to receive treatment than non-minorities (OR for treatment $0.54,95 \%$ CI 1.22 to 3.38 ). People aged 75 years or over were significantly less likely to receive treatment than people younger than 70 years $(75-79$ years age group OR $0.66,95 \%$ CI 0.46 to $0.97 ; \geqslant 80$ years age group OR $0.44,95 \%$ CI 0.32 to 0.60 ). People with supplemental insurance were

For correspondence: Dr S Crystal, Institute of Health, Health Care Policy, and Aging Research, Rutgers University, New Brunswick, New Jersey, USA; scrystal@rci.rutgers.edu

Sources of funding: funded in part by the Public Health Services Grants. more likely to be diagnosed with depression and to receive treatment than those without (OR for diagnosis $1.85,95 \%$ CI 1.41 to 2.42 ; OR for treatment $2.03,95 \%$ CI 1.22 to 3.38 ).

\section{CONCLUSIONS}

The incidence of depression in the elderly increased rapidly from 1992-98. Increasing age, belonging to a non-African American ethnic minority, and lack of supplemental insurance all reduced the likelihood of receiving treatment for depression among the elderly Medicare population.

\section{Commentary}

rystal et al's excellent paper provides us with the most comprehensive examination to date of treated prevalence of depression among the elderly. As such, it offers opportunity for celebration and cause for concern. First, the celebration: Crystal et al's results indirectly inform us about the clinical and social culture that surrounds diagnosis and treatment of depression among the elderly, suggesting that clinicians and their patients are becoming more comfortable with acknowledging and accepting diagnoses and treatment of depression. Such changes provide increased opportunities for addressing this serious problem in a population historically under diagnosed and under treated. These findings also provide an important indicator of treatment quality: those with major depressive disorder were more likely to receive treatment than those with less severe diagnoses, suggesting rational decision making. Greater probability of treatment given poorer health status is also good news, for it suggests that clinicians are taking opportunities associated with treating other health problems to identify cases of depression, and that they are not less likely to attend to, or identify, depressive symptoms in the face of multiple comorbidities.

This paper also highlights significant areas for concern. Although African Americans were as likely as whites to receive depression treatment, other disadvantaged groups did not fare as well. Those of "Hispanic or other" ethnicity and the oldest old were less likely to receive treatment when diagnosed. Of even greater concern are findings indicating that limited insurance coverage reduces the likelihood of diagnosis, and of treatment given diagnosis. Clinicians should be alert to the fact that elderly individuals with limited coverage (Medicare alone), the oldest old, and members of some ethnic groups may be less likely to avail themselves of treatment, even when prescribed. Working with these individuals to identify and address barriers to treatment could significantly improve care for these disadvantaged individuals.

Carla A Green, PhD MPH

Oregon Health \& Science University and Kaiser Permanente Center for Health Research, Portland, Oregon, USA 